\title{
Ontologies for Production Automation
}

\author{
Jose L. Martinez Lastra and Ivan M. Delamer \\ jose.lastra@tut.fi, ivan.delamer@ut.fi
}

\begin{abstract}
The manufacturing sector is currently under pressures to swiftly accommodate new products by quickly setting up new factories or retrofitting existing ones. In order to achieve this goal, engineering tasks currently performed manually need to be automated. In this scenario, ontologies emerge as a candidate solution for representing knowledge about manufacturing processes, equipment, and products in a machine-interpretable way. This knowledge can then be used by automated problem-solving methods to (re)configure the control software that coordinates and supervises manufacturing systems. This chapter reviews current approaches to use ontologies in the manufacturing domain, which include use for vocabulary definition in multi-agent systems and use for describing processes using Semantic Web Services. In addition, current and emerging research trends are identified.
\end{abstract}

\section{Introduction}

As in many other domains, the pervasiveness of information and embedded computational power is causing a revolution in the way manufacturing systems are conceived. The drivers for such a revolution vary in different markets:

- In the high-tech and consumer product markets, the drivers are increased customization of product, higher new product introduction rates, and shorter time to market requirements.

- In other discrete product manufacturing markets, the drivers are reduction in lead time and supply chain responsiveness.

- In process and batch manufacturing markets, such as (petro)chemical, paper, mining, and so on, the drivers are increased quality and reduced factory downtime requirements.

- In developed markets, the drivers are improving competitiveness against economies of scale and emerging markets.

- In developing markets, the drivers are production in proximity to the target markets, and competition against established markets.

In order to respond to these requirements and remain competitive, manufacturing enterprises must be able to swiftly set up new factories, and more importantly to seamlessly re-configure existing factories in order to produce new product variants or new product families. However, these tasks require significant amounts of engineering efforts, which result in time delays in the order of months or even years, and major expenditures in highly-qualified engineers. 
One of the main reasons why engineering efforts are needed is that technical specifications for products, for the processes required to manufacture them, and for the devices and machines that are used are written in natural language, often aided by visual diagrams. These specifications are combined with the pre-existing know-how and experience of engineers to systematically develop the physical configuration of the factory and the automatic control programs that coordinate and supervise the processes. Whenever changes are needed to accommodate a new product or process, engineers are brought in to transform the specifications coming from the product design and the existing system into a modified manufacturing system.

In order to overcome most or all of the tasks that are currently performed manually by engineers on a case-by-case basis, the knowledge contained in technical specifications and engineering know-how must be given a machine-interpretable form, so that automated problem-solving methods can be used to (re-)configure systems. Ontologies have recently emerged as a strong candidate to represent the many different sources of knowledge used in production automation in a modular and reusable approach.

The rest of this chapter is an attempt to describe what types of knowledge need to be represented in order to facilitate the (re-)configuration of manufacturing systems, and what approaches are currently being followed in order to represent this knowledge using ontologies. The next subsection presents some background information about the manufacturing domain. Subsequently, the main domains of knowledge representation are introduced: processes, products, and equipment. The latter part of this chapter is then dedicated to explore how different researches have attacked the problem and what research directions are currently emerging.

\section{Characteristics of Manufacturing Systems}

Manufacturing systems can be coarsely defined to be composed by a series of processing station and by a material handling system that transfers and supplies products, parts, components and materials to the stations as required for each product variant being manufactured. Traditional manufacturing systems were composed of a linear process flow, called a transfer line, which transfers products through a fixed sequence of processing station, each of which perform a pre-defined process. Such transfer lines are usually highly efficient ${ }^{1}$, but inflexible in that they are usually capable of producing only one type of product and require significant effort and down-time to be reconfigured for a new type of product. Thus, many newer manufacturing systems are composed of a flexible material handling system, which is able to provide multiple material routing paths and therefore many possible processing sequences, and of flexible and/or reconfigurable process cells.

Flexible production systems emerged during the 1980s, facilitated by an increase in availability of programmable robotized cells (used mostly for assembly and painting) and of programmable numerically-controlled machines (used for machining parts). Flexible process cells are able to perform a wide array of processes, therefore being

\footnotetext{
${ }^{1}$ Transfer lines are usually efficient while online, although they are less robust in case of breakdowns as the whole line needs to be stopped.
} 
able to cope with a wide variety of products over its lifetime without need for altering or retrofitting the equipment. However, this flexibility implies that the equipment is typically underutilized, causing the excess capabilities to be an unwanted capital investment. As a result, reconfigurable process cells have gained attention as a paradigm that fosters the adoption of equipment with interchangeable parts, tools and mechatronic actuators that can be easily composed in different configuration according to the product requirements. Reconfigurable process cells meet without exceeding the process requirements for the products being manufactured, and can easily be changed or reconfigured when new products are introduced [1].

In both the cases where flexible or reconfigurable process cells are used, a number of mechatronic devices/modules/subsystems are coordinated to implement the process offered by the machine. These devices may include conveyors, fixturing actuators, articulated robot arms, tools such as screwdrivers, welding electrodes, drills, etc, and/or end effectors such as grippers, among others. Each device provides one or more physical operations, which can be coordinated with other devices' operations to implement complex processes, e.g. a conveyor, robot arm and gripper operations can be coordinated to implement a pick\&place machine. Likewise, at a higher hierarchical level, the processes offered by the different stations can be sequenced in order to yield different products. Therefore, low-level physical operations can be coordinated to output different manufacturing processes, and these processes can then be sequenced to yield different types of products.

The currently available collection of industrial devices that are used to develop manufacturing equipment is extremely large. Likewise, the catalog of industrial equipment that can be used to generate different manufacturing systems is a large volume. However, the physical operations and manufacturing processes provided by the devices and equipment is a much more reduced set, which also changes slowly over time (even if frequent innovations cause the equipment to implement those processes in new ways). Therefore, production engineers are able to select appropriate devices/machines according to case-specific specifications based on their previous knowledge and experience on the required operations/processes.

This suggests that if existing knowledge on physical operations and manufacturing processes is represented in a machine-interpretable format, many of the (time- and resource-costly) design and configuration tasks that are currently performed by engineers could become more automatic. In this scenario, ontologies emerge as a candidate solution to represent knowledge about operations and processes, and about devices and equipment that offer those operations/processes. Such a representation would allow different reasoning mechanisms to be applied, such as subsumption for classifying new types of devices/equipment, or more complex problem-solving methods for composition and configuration purposes.

Furthermore, ontologies can be used to represent product knowledge. Such knowledge can be used during product design in order to create configurations or compositions of parts, sub-assemblies and components. However, the focus in this chapter shall be on the use of product knowledge in order to derive the process requirements that are used to develop the corresponding manufacturing system. 


\section{Process, Product and Equipment Ontologies}

The first thing that is realized when developing an ontology for the manufacturing domain is that the common concept that is involved in all major portions of knowledge specification is the process. From a manufacturing equipment perspective, the main purpose or function is to offer a process. From a product perspective, the main requirement is a set of processes used to transform parts and raw materials into finished goods. The relationship between these major concepts is illustrated in Fig. 1.

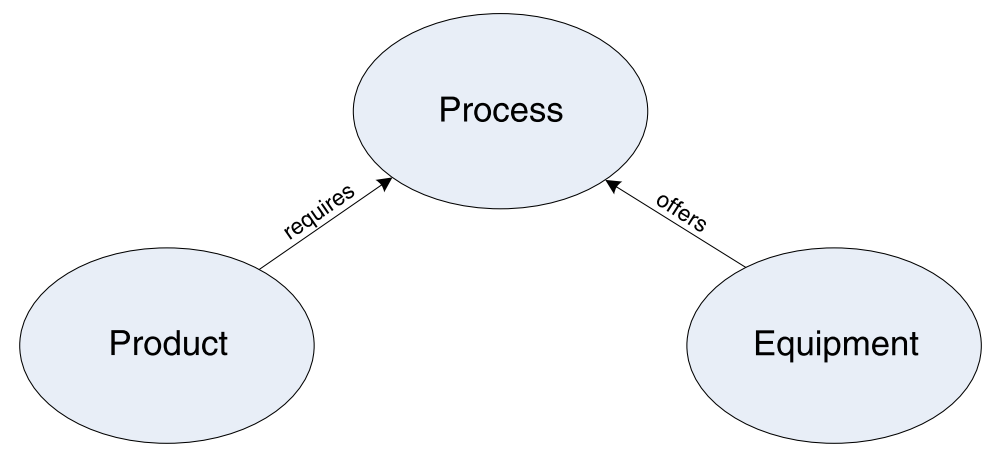

Fig. 1. Upper concepts in a manufacturing ontology

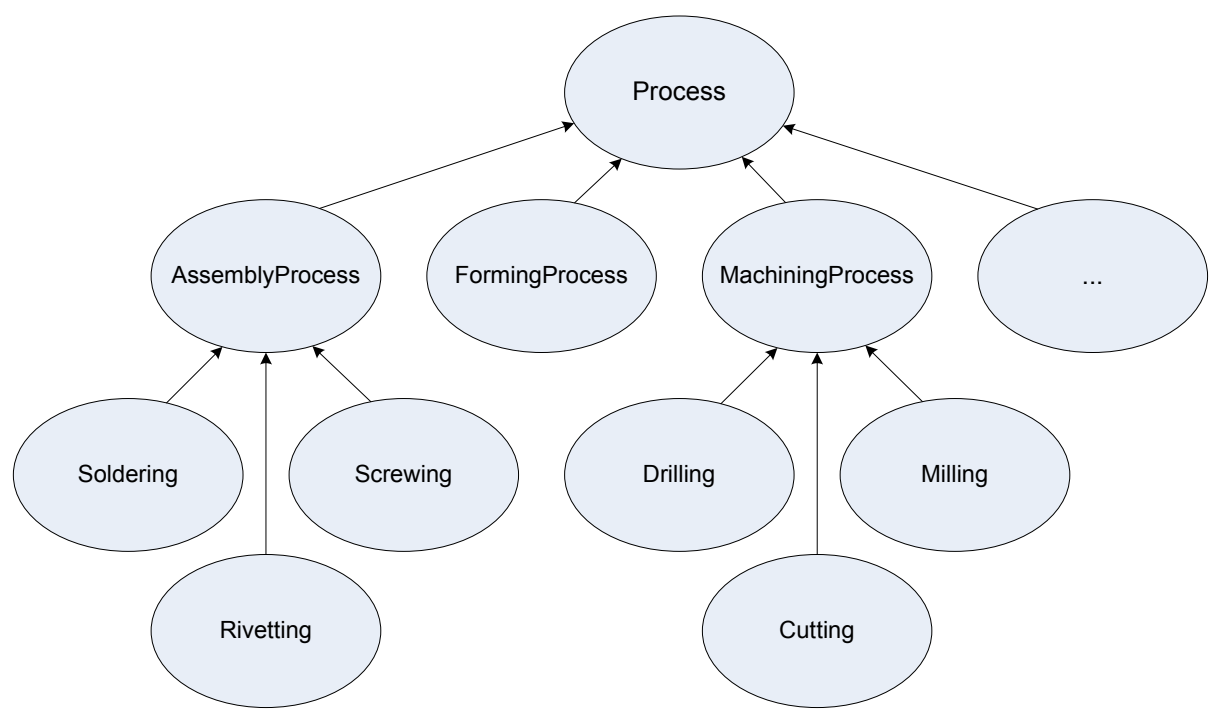

Fig. 2. A sample process taxonomy

\subsection{Process Taxonomies}

The three upper concepts, Process, Equipment and Product, typically serve as base concepts that are refined by sub-concepts representing more specific types of processes, 
equipment, and products. While the classification of equipment and products can be performed in numerous ways, processes are typically represented using a hierarchical taxonomy. This taxonomy can then be used to match required processes (from products) to offered processes (by equipment). A sample process taxonomy is illustrated in Fig. 2.

\subsection{Product Ontologies}

Conceptual specifications of products typically include both geometric information and non-geometric information. The hierarchical classification of concepts varies, and some ontologies may even place the same concepts in several parallel tree-like structures, e.g. according to product function, geometric information, product market type, required manufacturing processes, etc. Regardless of the adopted hierarchy model, certain product concepts that are specified in ontologies are predominant.

For assembled products, conceptualizations include parts and connections between parts. Parts may be atomic components or subassemblies, which are modular assemblies of components which can then be assembled to other parts. Different types of connection concepts can be linked to process concepts from a process taxonomy, allowing to infer which process is needed to connect or assembly two parts. In addition to connection concepts, non-geometric information that is typically added to the product concept includes manipulation information, storage information, and other lifecycle-related information. An overview of concepts related to assembled products is illustrated in Fig. 3.

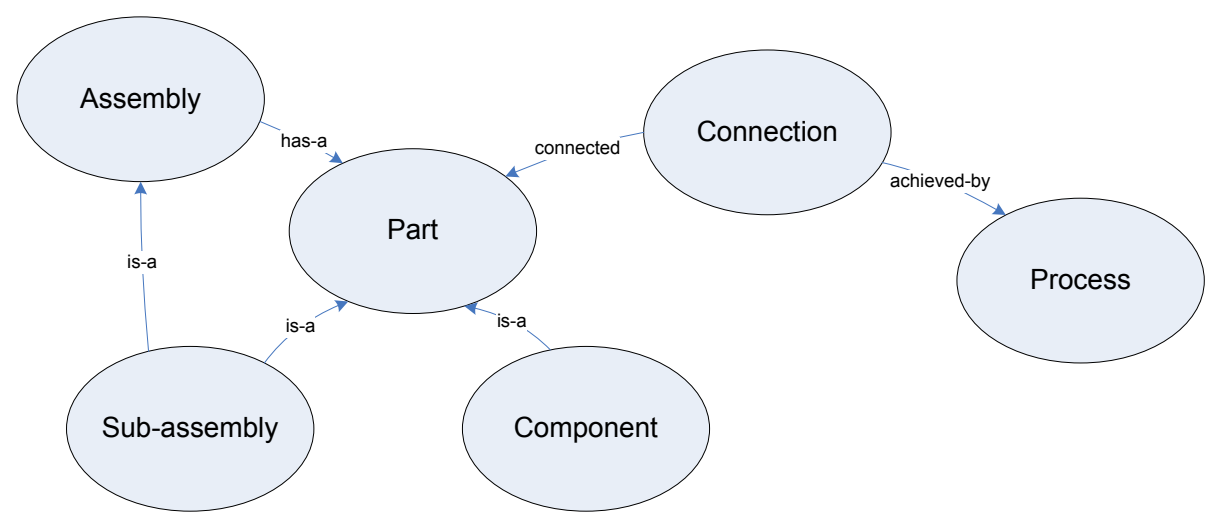

Fig. 3. Concepts typically found in ontologies for assembled products

For most metal products, machining processes such as cutting, drilling, and milling are used to remove material from a base cast part until the desired geometry is achieved. The specification of the product is usually achieved by composing primitive geometric shapes (such as cubes, cylinders, etc) which may be either filled (indicating the shape of the part) or blank (indicating where material is removed). In particular where material is removed, non-geometric information is included to indicate which types of processes can be used to achieve the geometry. For example, an empty cylinder may be associated to a drilling process, which is specified in a process taxonomy. 
For virtually any type of product, traditional data structures for product representation have relied solely on 3D geometric data acquired using CAD software. The conceptualizations used in ontologies allow incorporating non-geometric information (in the form of connections, associated processes, etc) that represents the implicit knowledge that is traditionally held by engineers, but seldom conveyed with product specifications. This has often resulted in a knowledge gap between design engineers and manufacturing engineers, who need to figure out much of the semantic content that was left implicit during product design in order to develop a manufacturing system.

\subsection{Equipment Ontologies}

Traditionally, machines used in manufacturing were large, monolithic pieces of equipment. The drivers for flexibility and reconfiguration as well as time and cost pressures have led to developing machines from modular subsystems and mechatronic devices which provide different operations. An insight that is normally incorporated into modular equipment ontologies is that, like an assembled product, a machine is an assembly of devices. Therefore, similar structures as those used for representing products can be used to represent machines, utilizing geometric information and nongeometric information such as connections between modules and information about the physical operations facilitated by the device.

However, the unique feature of an equipment ontology is that an account must be given for the notion of amplification of skills: the composition of the atomic skills of devices to create more complex processes. As an example, the composition of vertical displacement and torque application results in a screw-driving process. Whenever amplification of skills occur, the resulting equipment is able to offer a process that is in addition to the atomic operations provided by the individual devices.

An equipment ontology will thus contain concepts for particular types of devices and hardware modules, and will also contain concepts corresponding to different types of machines and how these are composed. Therefore, by analyzing the properties of a particular configuration of devices and hardware modules, it is possible to classify a machine and determine what types of manufacturing processes it is able to offer.

\subsection{Refined Process Ontologies}

While a process taxonomy is sufficient to conceptualize the operations required to manufacture a product, and to analyze the configuration of a piece of equipment and infer what processes it may offer, a taxonomy is not sufficient to model the dynamic nature of processes. A taxonomy will suffice if all that is necessary is for software agents to model a manufacturing environment, infer the skills of a machine and determine the requirements of a product. However, it is not enough for software agents to dynamically evolve this model as processes occur. Therefore, process taxonomies are often either extended or complemented by an additional ontology in order to model the dynamic behavior observed before, during and after the execution of a process.

A refined process ontology will specify aspects such as:

- The states of the products and equipment in which the process can be executed.

- The coordination of operations needed to complete a process.

- The resulting state of the products and equipment after the process is completed. 
Thus, agents are able to infer whether a required process can be achieved (or the associated capability to plan a set of actions that will lead to a valid state), can automatically coordinate the execution of operations and processes, and can evolve their internal representation of the physical environment to the resulting state.

\subsection{Auxiliary Ontologies}

In addition, some auxiliary ontologies are typically used in order to specify the concepts related to processes, equipment and products. These ontologies are typically developed so that they can be reused across domains, and are not specific to the manufacturing environment. The most commonly used auxiliary ontologies are used for conceptualizing:

- Geometry, such as shapes and volumes of parts, products, mechanical devices, and so on.

- Physical units, such as units of length, volume, temperature, force, pressure, velocity, acceleration, and so on.

- Software interfaces, used for invoking devices and machines.

\section{Brief Survey and State of the Art}

The use of ontologies in the manufacturing domain has evolved over the last 10 years starting since the inception of the Process Specification Language (PSL), developed by NIST [2]. Although it never gained a lasting acceptance, PSL would come around to influence several latter approaches, most notably those based on Semantic Web Services. Ontologies began to find its place in manufacturing automation architectures as a part of larger efforts focusing on multi-agent systems for manufacturing control. The use of ontologies has since been heavily influenced by developments outside the manufacturing domain, most notably the emergence of Web-based solutions and the Semantic Web.

This chapter section reviews some of the most prominent applications of ontologies in the manufacturing domain to date. The approaches can be classified into three groups:

- Multi-agent systems, which utilize ontologies as a shared vocabulary for interagent communications for production planning and scheduling.

- Design support systems, which utilize ontologies to guide the design process of manufacturing systems.

- Service-oriented systems, in which equipment offer processes as Web Services that are described by ontologies. Process-oriented Semantic Web Services can then be dynamically discovered, selected, composed and invoked.

\subsection{Ontologies and Multi-agent Systems}

During the late 1990's and early 2000's, the Holonic Manufacturing Systems (HMS) consortium merged a large portion of the research done on the application of multiagent systems for manufacturing control. The initiative targeted the development of 
systems composed of modular and autonomous units, called holons, which would be able to self-organize and automate the control of the manufacturing system through the emergent behavior of a large group of holons. This target led to the adoption of multi-agent technologies developed in other domains, with particular emphasis on utilizing FIPA (Foundation for Intelligent Physical Agents) standards ${ }^{2}$. The body of work resulting from the HMS initiative has been very influential to the point that many recent developments continue to use the same technologies (e.g. KIF ontologies) in order to provide backwards compatibility.

Within the realm of multi-agent systems, both those developed under HMS and under other initiatives, ontologies have been mainly used as a means to provide a shared vocabulary that agents can use to construct messages and conversations [3] [4]. Following the original FIPA specifications, these ontologies were defined using the Knowledge Interchange Format (KIF). More recent developments have explored utilizing Web Ontology Language (OWL) ontologies, as well as utilizing ontology reconciliation techniques in order to enable interoperability among agents that use different ontologies [5].

An example ontology that is used in a multi-agent context is the Manufacturing Ontology in the ADACOR (ADAptive holonic COntrol aRchitecture for distributed manufacturing systems) Architecture [6], which is illustrated in Fig. 4. The ontology follows the Frame (F-Logic) formalism, as suggested by the FIPA standards. The main purpose of the architecture is to dynamically schedule and dispatch work orders, which result in material being sent to the appropriate process stations. The scope of the ontology is to support such scheduling functions.

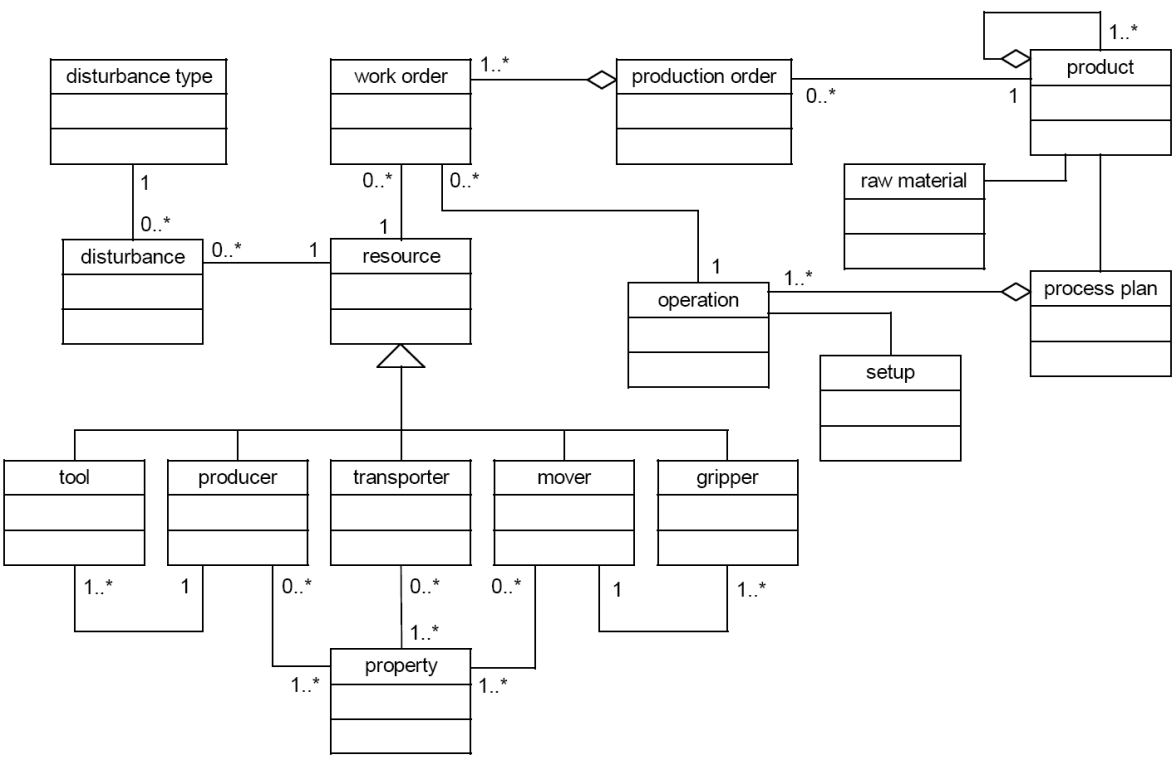

Fig. 4. Upper concepts of the manufacturing ontology in the ADACOR Architecture

\footnotetext{
${ }^{2}$ www.fipa.org
} 


\subsection{Ontologies for Design Support}

As an intermediate solution towards automatic (re)configuration of manufacturing systems, the use of design tools that guide or suggest solutions through the design process can significantly reduce the required efforts. Inference mechanisms can then be used to rank and suggest alternative solutions on how to develop manufacturing equipment based on product requirements.

One such approach is proposed in [7], who developed a Web-based system that is used to develop assembly cells from mechatronic modules. The work reports an ontology framework for describing products, processes used for product assembly, and equipment that implement those assembly processes. The interactive Web-based system utilizes these ontologies in the background in order to guide the designer, starting from the product definition, in selecting devices/modules and their physical configuration that is required for the assembly the given product. The ontologies are specified using the Protégé frame-based language and following the design principles originally put forward in the CommonKADS methodology [8].

Although a big step forward in terms of facilitating the (re-)configuration of modular assembly systems, current design support solutions operate on functional and mechanical knowledge, i.e. the operations/processes provided by each module and how to mechanically attach them. However, the automatic control of the assembly cell is not considered, and must still be performed manually by control engineers once the modules are physically configured.

\subsection{Semantic Web Services in Factory Automation}

One of the shortcomings of existing approaches to manufacturing control is that the interactions between distributed control software components must be programmed manually according to the types of interfaces offered by the equipment to be controlled. The multi-agent approach attempts to overcome this problem by implementing several types of interactions aimed at covering the wide spectrum of systems. However, when new types of processes or devices are introduced into a system, existing control software elements (such as agents) must be re-programmed to be able to interact with the novel devices.

What is desirable is to have devices expose and describe their interfaces in a way that control software elements can dynamically acquire sufficient knowledge to interact with those devices. This notion has led to the adoption of Service-Oriented Architecture (SOA) for devices to dynamically publish and locate processes encapsulated as Web Services, and to the use of ontologies to describe those Web Services. Thus, control software entities such as software agents can dynamically discover available Web Services and infer sufficient knowledge on how to invoke and compose the underlying physical processes.

This is the approach that was originally reported in [9] and is currently being put forward in a number of European projects, among them SOCRADES ${ }^{3}$ (Serviceoriented Cross-layer Architecture for Distributed smart Embedded Devices), and SODA $^{4}$ (Service-oriented Device Architecture). The approach adopts technologies

\footnotetext{
${ }^{3}$ www.socrades.eu

${ }^{4}$ www.soda-itea.org
} 
from the Web Services and Semantic Web domains in order to maximize interoperability. A Semantic Web Services ontology, such as OWL-S [10], is then used to describe the services offered by equipment in a way that they can be dynamically discovered, selected, composed and orchestrated.

In the work reported in [9], the process taxonomy is based on the DIN 8593 standard [11], which specifies and classifies joining processes, and which has been specified as an OWL ontology. In addition, it follows the previous work of Vos [12] who identified the basic physical operations that are needed in order to achieve certain assembly processes. Thus, the joining processes in the taxonomy (such as screwing, press-fitting, etc) are linked to atomic physical operations (such as forces, torques, displacements, etc). A sample view of the process taxonomy is given in Fig. 5 .

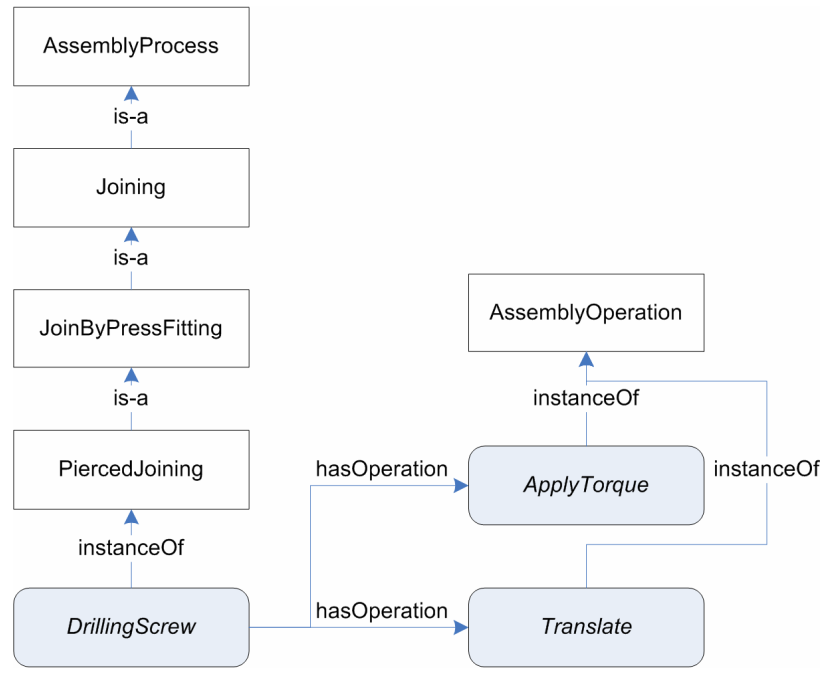

Fig. 5. Sample view of the process taxonomy

The product ontology is based heavily on the concept of assembly features, which is used to describe geometric and non-geometric (functional) information about how product parts are connected. Assembly features were originally proposed by Holland [13] and have been specified in an OWL ontology. Thus, a Connection Feature concept holds information about the geometry of the connection of the two parts, and the type of joint. The type of joint (e.g. a screw) can then be linked to a process (e.g. screwing) from the process taxonomy in order to dynamically define the required processes.

The equipment ontology is based on the Actor Based Assembly Systems (ABAS) reference architecture, reported by Martinez Lastra [14]. In this architecture, atomic mechatronic devices called Actors provide one of the atomic operations represented in the taxonomy. The Actors can then be grouped into Clusters in order to implement composite assembly processes, for example a vertical translation actor and a torque actor can be combined into a Screwing cluster. Clusters are modeled as an assembly of actors, using the same types of assembly features used for describing products. The properties of the upper Actor, Cluster and related concepts are illustrated in Fig. 6. 


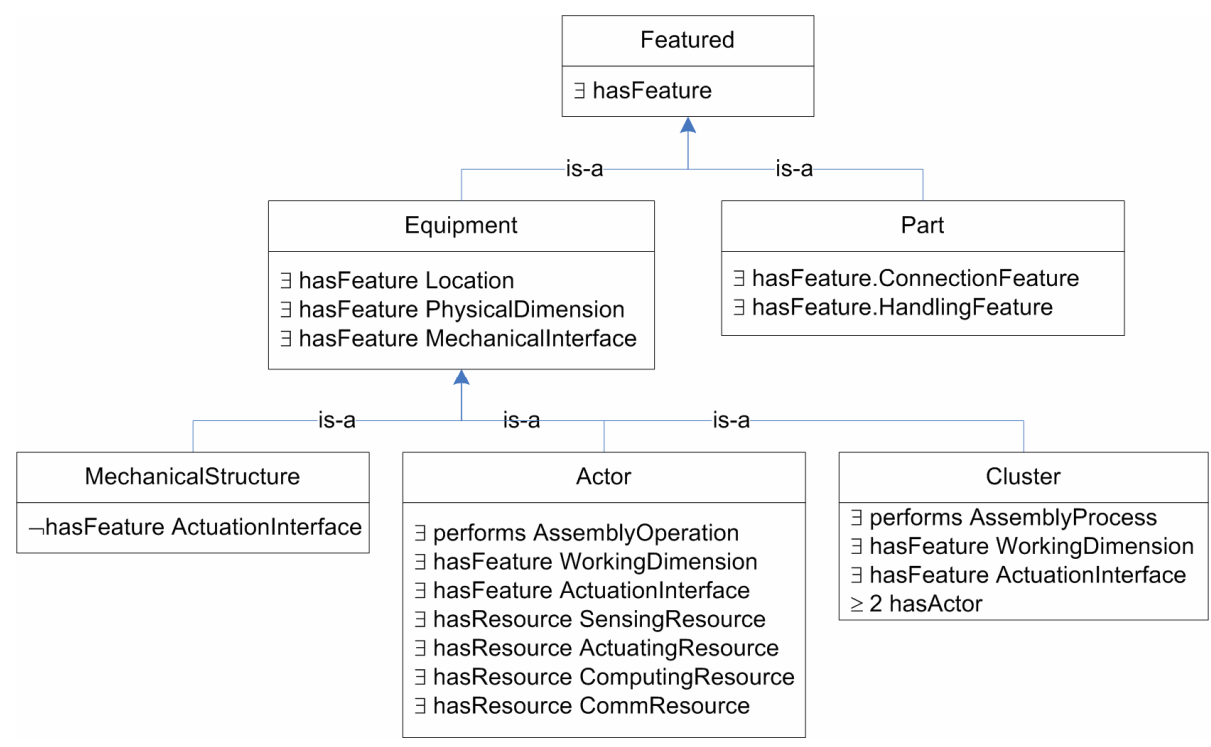

Fig. 6. Summary of Equipment upper concepts and properties

The process taxonomy, equipment ontology and product ontology offer a static model of the system that allows automatically inferring:

- The skills of Actors (mechatronic devices)

- The combined skills of Clusters (assembly cells)

- The required processes to assemble a product

- The clusters that are able to perform the required processes (matchmaking).

However, this static view is not sufficient for intelligent software agents to infer how the equipment should be used, i.e. how to invoke its processes in a changing environment. For this reason, a description is needed for:

- The software interface that allows invoking the process.

- The states of the environment in which the process can be invoked, i.e. the states of the product and of the equipment in which the process can be successfully executed.

- The resulting states of the environment after the process is executed, i.e. the resulting state of the product and of the equipment.

For this purpose, the OWL-S upper ontology for Web Services is used [10]. In particular, the Service Profile is used to model how operations are orchestrated to implement processes. Also, Preconditions and Effects are used to model the initial and consequent states of the world upon execution of the process. An example effect is the creation of a Connection Feature instance after a joining process is invoked (e.g. after a screwing process).

Compared to the inferences enabled by the static model offered by the process taxonomy, equipment ontology and product ontology, the addition of the dynamic model using a Semantic Web Service upper ontology allows the following tasks to be automated: 
- The coordination or orchestration of Web Services that encapsulate operations in order to achieve higher-level processes.

- The generation of the necessary messages to invoke a Web Service (and its underlying physical process).

- The validation of preconditions necessary for the invocation of the process. Preconditions also provide a target state that can be used for planning in the case that the conditions are not immediately met.

- The evolution of the model of the product and of the equipment, according to the effects of the Web Service (and its underlying physical process).

As a result of the knowledge contained in all ontologies, intelligent agents are able to derive the specification of required processes for a product, to discover available equipment and select the appropriate devices according to the required processes, and to invoke those devices. The relationship of the different ontologies used is illustrated in Fig. 7.

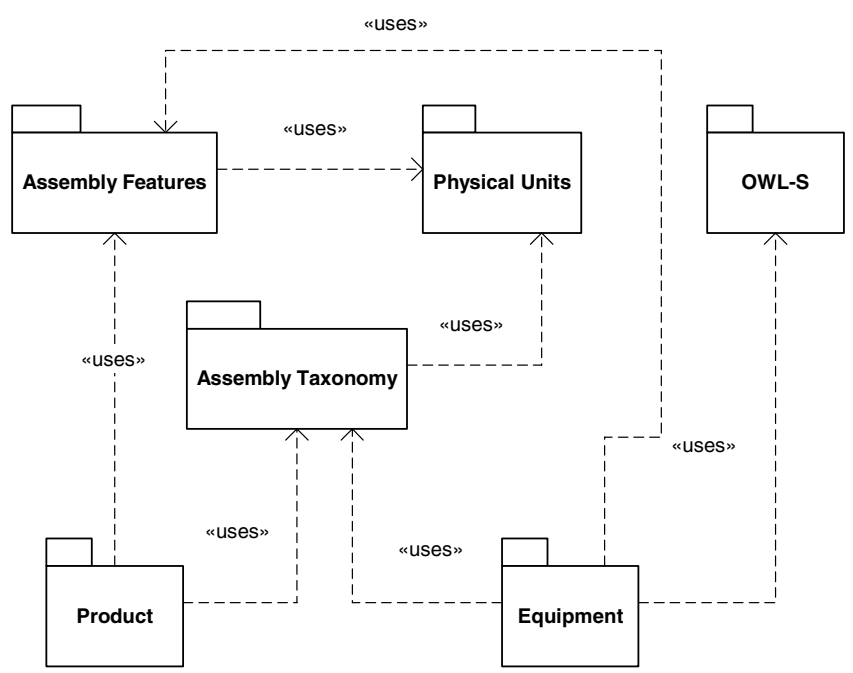

Fig. 7. Relationship between ontologies used for automation of assembly processes

\subsection{State of the Art summary}

Ontologies are currently the state-of-the-art technology in the manufacturing domain for the following purposes:

- Enabling interoperability of autonomous agents, specifically in the realm of dynamic process planning and scheduling where agent societies use ontologies to define a shared vocabulary in communications.

- For manufacturing equipment design support

- Dynamic system (re-)configuration using domain ontologies to derive required processes and Semantic Web Services to describe and encapsulate offered processes. 


\section{Emerging Research Directions}

The experiences that have reported use of ontologies have generated interest into further developing the frameworks, infrastructures and architectures that have proposed their use. Some of the ongoing and emerging research directions targeting the use of ontologies in the manufacturing domain include:

- Enhance process/task ontologies to include notions of time. The modeling of the time taken to execute a process is currently missing or insufficient in most ontologies, which makes it more difficult to autonomously plan optimal process sequences.

- Use First-Order Logic task ontologies. Current approaches use Description Logics and struggle somewhat to describe dynamic aspects of the processes (such as preconditions and effects).

- Optimized algorithms for dynamic A-Boxes. Most inference engines are optimized for incremental additions of knowledge, and not for revision of knowledge (e.g. when the state of a product or equipment changes).

- Application of ontology reconciliation algorithms. Most current approaches operate only with shared ontologies and fail when different ontologies need to be used together (e.g. if devices from different vendors are used together).

- Ontology mediation. As an alternative to ontology reconciliation, mediators could be used to automatically translate between ontologies.

- Consolidation of ontologies and development of ontologies for specific domains.

- Pilots and demonstrations. Further demonstrators are needed to illustrate the application of ontologies, to show the feasibility of the approach, and to facilitate technology transfer to industry.

- Applications to diagnostics. Factories produce a constant stream of process data that reflects innumerable conditions in the factory, and that needs to be interpreted in real time. Ontologies may assist in modeling the relationships of data sources, modeling conditions and events, and facilitating reasoning that leads to diagnosis of caution and/or error conditions.

- Applications to enterprise integration. Ontologies have found extensive application to the domain of Enterprise Information Systems, and ongoing research is targeting at using ontologies to facilitate the integration of Enterprise and Factory Information Systems.

\section{Conclusions and Outlook}

Current market trends are driving manufacturers to set up and/or reconfigure their production systems faster and at lower costs. The engineering efforts that are currently invested need to be replaced by automation of (re)configuration tasks. In this scenario, ontologies emerge as the state-of-the-art mechanism to represent manufacturing domain knowledge in a modular and reusable approach - the representation of knowledge is a prerequisite for applying automated problem-solving methods.

Ontologies are being applied as part of large research initiatives involving prominent members of both academia and industry, with significant investments planned for the coming years. As ontologies continue to expand in other domains, the maturity 
of these related approaches will have a significant impact in the manufacturing domain, as is currently observed with the use of Semantic Web Services.

\section{References}

1. Mehrabi, M.G., Ulsoy, A.G., Koren, Y.: Reconfigurable manufacturing systems: Key to future manufacturing. Journal of Intelligent Manufacturing 11(4), 403-419 (2000)

2. Schlenoff, C., Grüninger, M., Tissot, F., Valois, J., Lubell, J., Lee, J.: The Process Specification Language (PSL): Overview and Version 1.0 Specification. NISTIR 6459, National Institute of Standards and Technology, Gaithersburg, MD (2000)

3. Foundation for Intelligent Physical Agents: FIPA Ontology Service Specification Geneva, Switzerland (2001), http: / /www.fipa.org/specs/fipa00086/XC00086D.html

4. Obitko, M., Marik, V.: Ontologies for Multi-Agent Systems in Manufacturing Domain. In: Hameurlain, A., Cicchetti, R., Traunmüller, R. (eds.) DEXA 2002. LNCS, vol. 2453, Springer, Heidelberg (2002)

5. Obitko, M., Mařík, V.: Integrating Transportation Ontologies. In: Mařík, V., William Brennan, R., Pěchouček, M. (eds.) HoloMAS 2005. LNCS (LNAI), vol. 3593, pp. 99-110. Springer, Heidelberg (2005)

6. Borgo, S., Leitao, P.: Foundations for a core Ontology of Manufacturing. In: Kishore, R., Ramesh, R., Sharman, R. (eds.) Ontologies: A Handbook of Principles, Concepts and Applications in Information Systems. Springer, Heidelberg

7. Lohse, N.: Towards an Ontology Framework for the Integrated Design of Modular Assembly Systems. Doctoral Thesis, University of Nottingham (2006)

8. Schreiber, G., Wielinga, B., de Hoog, R., Akkermans, H., Van de Velde, W.: CommonKADS: A Comprehensive Methodology for KBS Development. IEEE Expert, 28-37 (December 1994)

9. Delamer, I.M.: Event-based Middleware for Reconfigurable Manufacturing Systems: A Semantic Web Services Approach. Doctoral dissertation No. 631, Tampere University of Texhnology, Finland (2006) ISBN 952-15-1672-0

10. The OWL Services Coalition: OWL-S: Semantic Markup for Web Services. DAML-S Home Page (2007), http: / /www. daml . org / services / owl-s /

11. Deutsches Institute für Normung e.V., Manufacturing Processes Joining, DIN 8593 Parts 0-8 (2003) (in German)

12. Vos, J.A.W.M.: Module and System Design in Flexibly Automated Assembly. Ph.D. Thesis, Delft University of Technology, The Netherlands (2001)

13. Van Holland, W., Bronsvoort, W.F.: Assembly Features in modeling and planning. Robotics and Computer Integrated Manufacturing 16, 277-294 (2000)

14. Martinez Lastra, J.L.: Reference Mechatronic Architecture for Actor-based Assembly Systems. Doctoral dissertation No. 484, Tampere University of Technology, Finland (2004) ISBN 952-15-1210-5 